\title{
HANKEL DETERMINANT OF SECOND ORDER FOR SOME CLASSES OF ANALYTIC FUNCTIONS
}

\author{
Milutin Obradović ${ }^{1}$ and Nikola Tuneski ${ }^{2, *}$ \\ ${ }^{1}$ Department of Mathematics, Faculty of Civil Engineering, University of Belgrade, Bulevar Kralja Aleksandra \\ 73, 11000, Belgrade, Serbia \\ ${ }^{2}$ Department of Mathematics and Informatics, Faculty of Mechanical Engineering, Ss. Cyril and Methodius \\ University in Skopje, Karpoš II b.b., 1000 Skopje, Republic of North Macedonia \\ Communicated by László Tóth \\ Original Research Paper \\ Received: Jan 25, 2021 - Accepted: Mar 14, 2021 \\ First published online: October 6, 2021 \\ (c) 2021 The Author(s)

\section{ABSTRACT} \\ Let $f$ be analytic in the unit disk $\mathbb{D}$ and normalized so that $f(z)=z+a_{2} z^{2}+a_{3} z^{3}+\cdots$. In this paper, we give upper bounds of \\ the Hankel determinant of second order for the classes of starlike functions of order $\alpha$, Ozaki close-to-convex functions and \\ two other classes of analytic functions. Some of the estimates are sharp.
}

\section{KEYWORDS}

analytic, univalent, Hankel determinant, starlike of order $\alpha$, Ozaki close-to-convex functions

\section{MATHEMATICS SUBJECT CLASSIFICATION (2020)}

$30 \mathrm{C} 45,30 \mathrm{C} 50$

\section{INTRODUCTION AND PRELIMINARIES}

Let $\mathcal{A}$ denote the family of all analytic functions in the unit disk $\mathbb{D}:=\{z \in \mathbb{C}:|z|<1\}$ satisfying the normalization $f(0)=0=f^{\prime}(0)-1$.

A function $f \in \mathcal{A}$ is said to be starlike of $\operatorname{order} \alpha, 0 \leq \alpha<1$, if, and only if

$$
\operatorname{Re}\left[\frac{z f^{\prime}(z)}{f(z)}\right]>\alpha \quad(z \in \mathbb{D}) .
$$

We denote this class by $S^{\star}(\alpha)$. If $\alpha=0$, then $S^{\star} \equiv S^{\star}(0)$ is the well-known class of starlike functions.

By $\mathcal{C}(\alpha),-\frac{1}{2} \leq \alpha<1$, we denote the class Ozaki close-to-convex functions consisting of functions $f \in \mathcal{A}$ for which

$$
\operatorname{Re}\left[1+\frac{z f^{\prime \prime}(z)}{f^{\prime}(z)}\right]>\alpha \quad(z \in \mathbb{D})
$$

\footnotetext{
*Corresponding author. E-mail: nikola.tuneski@mf.ukim.edu.mk
} 
The special case of this class, when $\alpha=-1 / 2$ was introduced by Ozaki in 1941 ([6]) and it is a subclass of the class of close-to-convex functions. This, general form of the class, was introduced in [3] by Kargar and Ebadian. We note that for $\alpha=0$ we have the class of convex functions.

More about this class one can find in [2] and [11].

Similarly, by $\mathcal{G}(\alpha) 0<\alpha \leq 1$, we denote the class of functions $f \in \mathcal{A}$ for which

$$
\operatorname{Re}\left[1+\frac{z f^{\prime \prime}(z)}{f^{\prime}(z)}\right]<1+\frac{1}{2} \alpha \quad(z \in \mathbb{D}) .
$$

Ozaki in [6] introduced the class $\mathcal{G}(1)$ and proved that functions in $\mathcal{G}(1)$ are univalent in the unit disk. Later, Umezawa in [12], Sakaguchi in [8] and R. Singh and S. Singh in [9] showed, respectively, that functions in $\mathcal{G}(1)$ are convex in one direction, close-to-convex and starlike.

Nunokawa in [4] considered the more general class $\mathcal{G}(\alpha)$ and proved that it is subclass of the class of strongly starlike functions of order $\alpha$, i.e., if $f \in \mathcal{G}(\alpha)$, then $\left|\arg \left[z f^{\prime}(z) / f(z)\right]\right|<\alpha \pi / 2$ for all $z \in \mathbb{D}$. This, general class is extensively studied by Obradović et al. in [5].

All previous mentioned classes are classes of univalent functions in the unit disc.

\section{MAIN RESULTS}

In this paper we will give the upper bound estimates for the Hankel determinant of second order for the previous given classes. Some of the estimates are sharp.

DEFINITION 2.1. Let $f \in \mathcal{A}$. Then the $q$ th Hankel determinant of $f$ is defined for $q \geq 1$, and $n \geq 1$ by

$$
H_{q}(n)=\left|\begin{array}{cccc}
a_{n} & a_{n+1} & \ldots & a_{n+q-1} \\
a_{n+1} & a_{n+2} & \ldots & a_{n+q} \\
\vdots & \vdots & & \vdots \\
a_{n+q-1} & a_{n+q} & \ldots & a_{n+2 q-2}
\end{array}\right| .
$$

Thus, the second Hankel determinant is $H_{2}(2)=a_{2} a_{4}-a_{3}^{2}$.

Namely, we have

THEOREM 2.2. Let $f(z)=z+a_{2} z^{2}+a_{3} z^{3}+\cdots$ belongs to the class $S^{\star}(\alpha), 0 \leq \alpha<1$. Then we have the next sharp estimation:

$$
\left|H_{2}(2)\right|=\left|a_{2} a_{4}-a_{3}^{2}\right| \leq(1-\alpha)^{2} .
$$

Proof. From the definition of the class $S^{\star}(\alpha)$, we have

$$
\frac{z f^{\prime}(z)}{f(z)}=\alpha+(1-\alpha) \frac{1+\omega(z)}{1-\omega(z)} \quad\left(=2 \alpha-1+2(1-\alpha) \frac{1}{1-\omega(z)}\right)
$$

where $\omega$ is analytic in $\mathbb{D}$ with $\omega(0)=0$ and $|\omega(z)|<1, z \in \mathbb{D}$.

From (2.1) we obtain

$$
f^{\prime}(z)=\left[1+2(1-\alpha)\left(\omega(z)+\omega^{2}(z)+\cdots\right)\right] \cdot \frac{f(z)}{z} .
$$

If we put $\omega(z)=c_{1} z+c_{2} z^{2}+\cdots$, and compare the coefficients on $z, z^{2}, z^{3}$ in the relation (2.2) then, after some calculations, we obtain

$$
\begin{aligned}
& a_{2}=2(1-\alpha) c_{1}, \\
& a_{3}=(1-\alpha)\left(c_{2}+(3-2 \alpha) c_{1}^{2}\right), \\
& a_{4}=\frac{2}{3}(1-\alpha)\left(c_{3}+(5-3 \alpha) c_{1} c_{2}+\left(2 \alpha^{2}-7 \alpha+6\right) c_{1}^{3}\right) .
\end{aligned}
$$

By using the relation (2.3), after some simple computations, we obtain

$$
H_{2}(2)=\frac{4}{3}(1-\alpha)^{2}\left(c_{1} c_{3}+\frac{1}{2} c_{1}^{2} c_{2}-\frac{1}{4}\left(4 \alpha^{2}-8 \alpha+3\right) c_{1}^{4}-\frac{3}{4} c_{2}^{2}\right) .
$$

From the last relation we have

$$
\left|H_{2}(2)\right| \leq \frac{4}{3}(1-\alpha)^{2}\left(\left|c_{1}\right|\left|c_{3}\right|+\frac{1}{2}\left|c_{1}\right|^{2}\left|c_{2}\right|+\frac{1}{4}\left|4 \alpha^{2}-8 \alpha+3\right|\left|c_{1}\right|^{4}+\frac{3}{4}\left|c_{2}\right|^{2}\right) .
$$


For the function $\omega(z)=c_{1} z+c_{2} z^{2}+\cdots$ (with $\left.|\omega(z)|<1, z \in \mathbb{D}\right)$ the next relations is valid (see, for example [7, expression (13) on page 128]):

$$
\left|c_{1}\right| \leq 1, \quad\left|c_{2}\right| \leq 1-\left|c_{1}\right|^{2}, \quad\left|c_{3}\left(1-\left|c_{1}\right|^{2}\right)+\overline{c_{1}} c_{2}^{2}\right| \leq\left(1-\left|c_{1}\right|^{2}\right)^{2}-\left|c_{2}\right|^{2} .
$$

We may suppose that $a_{2} \geq 0$, which implies that $c_{1} \geq 0$ and instead of relations (2.5) we have the next relations

$$
0 \leq c_{1} \leq 1, \quad\left|c_{2}\right| \leq 1-c_{1}^{2}, \quad\left|c_{3}\right| \leq 1-c_{1}^{2}-\frac{\left|c_{2}\right|^{2}}{1+c_{1}} .
$$

By using (2.6) for $c_{1}$ and $c_{3}$, from (2.4) we have

$$
\left|H_{2}(2)\right| \leq \frac{4}{3}(1-\alpha)^{2}\left[c_{1}\left(1-c_{1}^{2}\right)+\frac{3-c_{1}}{4\left(1+c_{1}\right)}\left|c_{2}\right|^{2}+\frac{1}{2} c_{1}^{2}\left|c_{2}\right|+\frac{1}{4}\left|4 \alpha^{2}-8 \alpha+3\right| c_{1}^{4}\right] .
$$

By using $\left|c_{2}\right| \leq 1-c_{1}^{2}$, from (2.7) after some calculations we obtain

$$
\left|H_{2}(2)\right| \leq \frac{4}{3}(1-\alpha)^{2}\left(\frac{3}{4}-\frac{3-\left|4 \alpha^{2}-8 \alpha+3\right|}{4} c_{1}^{4}\right) \leq(1-\alpha)^{2},
$$

since $3-\left|4 \alpha^{2}-8 \alpha+3\right| \geq 0$ for $0 \leq \alpha<1$. The equality in the last step is valid for $c_{1}=0$. The function $f_{\alpha}$, defined by the condition

$$
\frac{z f_{\alpha}^{\prime}(z)}{f_{\alpha}(z)}=\alpha+(1-\alpha) \frac{1+z^{2}}{1-z^{2}}
$$

(i.e where $\omega(z)=z^{2}, c_{2}=1$ and $c_{i}=0$ for $i \neq 2$ ) shows that the result of the theorem is sharp.

THEOREM 2.3. Let $f(z)=z+a_{2} z^{2}+a_{3} z^{3}+\cdots$ belongs to the class $\mathcal{C}(\alpha),-\frac{1}{2} \leq \alpha<1$. Then we have the next estimations:

$$
\left|H_{2}(2)\right| \leq \begin{cases}\frac{(1-\alpha)^{2}(5 \alpha+6)}{48(1+\alpha)}, & \text { if }-\frac{1}{2} \leq \alpha \leq 0 \\ \frac{(1-\alpha)^{2}\left(17 \alpha^{2}-36 \alpha+36\right)}{144\left(\alpha^{2}-2 \alpha+2\right)}, & \text { if } 0 \leq \alpha<1\end{cases}
$$

For $-\frac{1}{2} \leq \alpha \leq 0$ the result is sharp.

Proof. We will use the same method as in the proof of Theorem 2.2. From the definition of the class $\mathcal{C}(\alpha)$, similarly as in $(2.1)$ we have

$$
\left(z f^{\prime}(z)\right)^{\prime}=\left(1+2(1-\alpha)\left(\omega(z)+\omega^{2}(z)+\ldots\right)\right) f^{\prime}(z),
$$

where $\omega$ is analytic in $\mathbb{D}$ with $\omega(0)=0$ and $|\omega(z)|<1, z \in \mathbb{D}$.

If we put $\omega(z)=c_{1} z+c_{2} z^{2}+\cdots$, and compare the coefficients on $z, z^{2}, z^{3}$ in the relation (2.8) then, after some simple calculations, we obtain

$$
\begin{aligned}
& a_{2}=(1-\alpha) c_{1}, \\
& a_{3}=\frac{1}{3}(1-\alpha)\left[c_{2}+(3-2 \alpha) c_{1}^{2}\right], \\
& a_{4}=\frac{1}{6}(1-\alpha)\left[c_{3}+(5-3 \alpha) c_{1} c_{2}+\left(2 \alpha^{2}-7 \alpha+6\right) c_{1}^{3}\right] .
\end{aligned}
$$

Now, by using (2.9) we have, after some transformations,

$$
H_{2}(2)=\frac{1}{6}(1-\alpha)^{2}\left[c_{1} c_{3}+\frac{3-\alpha}{3} c_{1}^{2} c_{2}-\frac{1}{3}\left(2 \alpha^{2}-3 \alpha\right) c_{1}^{4}-\frac{2}{3} c_{2}^{2}\right]
$$

From the previous relation we have

$$
\left|H_{2}(2)\right| \leq \frac{1}{6}(1-\alpha)^{2}\left(\left|c_{1}\right|\left|c_{3}\right|+\frac{3-\alpha}{3}\left|c_{1}\right|^{2}\left|c_{2}\right|+\frac{1}{3}\left|2 \alpha^{2}-3 \alpha \| c_{1}\right|^{4}+\frac{2}{3}\left|c_{2}\right|^{2}\right) .
$$

As in the proof of Theorem 2.2, we may suppose that $c_{1} \geq 0$. In that case the relations (2.6) are valid and by using the inequality for $c_{3}$, from (2.11) we have

$$
\left|H_{2}(2)\right| \leq \frac{1}{6}(1-\alpha)^{2}\left(c_{1}\left(1-c_{1}^{2}\right)+\frac{2-c_{1}}{3\left(1+c_{1}\right)}\left|c_{2}\right|^{2}+\frac{3-\alpha}{3} c_{1}^{2}\left|c_{2}\right|+\frac{1}{3}\left|2 \alpha^{2}-3 \alpha\right| c_{1}^{4}\right) \text {. }
$$


From here, by using $\left|c_{2}\right| \leq 1-c_{1}^{2}$, we have (after some transformations):

$$
\left|H_{2}(2)\right| \leq \frac{1}{18}(1-\alpha)^{2}\left(2+(2-\alpha) c_{1}^{2}-\left(4-\alpha-\left|2 \alpha^{2}-3 \alpha\right|\right) c_{1}^{4}\right) .
$$

For $-\frac{1}{2} \leq \alpha \leq 0$, from (2.12) we obtain

$$
\left|H_{2}(2)\right| \leq \frac{1}{18}(1-\alpha)^{2}\left(2+(2-\alpha) c_{1}^{2}-2(1+\alpha)(2-\alpha) c_{1}^{4}\right) \leq \frac{(1-\alpha)^{2}(5 \alpha+6)}{48(1+\alpha)},
$$

because the function in the brackets attains its maximum for $c_{1}^{2}=\frac{1}{4(1+\alpha)}$.

The result is sharp if we choose $\omega(z)=z \frac{c_{1}-z}{1-c_{1} z}$ with $c_{1}=\frac{1}{2 \sqrt{1+\alpha}}$.

For the case when $0 \leq \alpha<1$ we use the same method.

\section{REMARK 2.4 .}

(i) Sokol and Thomas in [10] studied the second Hankel determinant for $\delta$-convex functions of order $\beta(\delta \in \mathbb{R}, 0 \leq \beta<1)$ of functions $f \in \mathcal{A}$ such that

$$
\left[(1-\delta) \frac{z f^{\prime}(z)}{f(z)}+\delta\left(1+\frac{z f^{\prime \prime}(z)}{f^{\prime}(z)}\right)\right]>\beta \quad(z \in \mathbb{D}),
$$

and for $\delta=0$ and $\delta=1$ received the same results as those given in Theorem 2.2 and Theorem 2.3.

(ii) As a special cases of Theorem 2.3, for $\alpha=-1 / 2$ and $\alpha=0$ we receive that for a function $f \in \mathcal{A}$, the following implications hold:

$$
\operatorname{Re}\left[1+\frac{z f^{\prime \prime}(z)}{f^{\prime}(z)}\right]>-\frac{1}{2} \quad(z \in \mathbb{D}) \quad \Rightarrow \quad\left|H_{2}(2)\right| \leq \frac{21}{64},
$$

and

$$
\operatorname{Re}\left[1+\frac{z f^{\prime \prime}(z)}{f^{\prime}(z)}\right]>0 \quad(z \in \mathbb{D}) \quad \Rightarrow \quad\left|H_{2}(2)\right| \leq \frac{1}{8} .
$$

The second implication is the same as the one in Theorem 4.2 .8 on page 63 from [11] where it is also shown that it os sharp.

THEOREM 2.5. Let $f(z)=z+a_{2} z^{2}+a_{3} z^{3}+\cdots$ belongs to the class $\mathcal{G}(\alpha), 0<\alpha \leq 1$. Then we have the next estimation:

The result is sharp for $\alpha=1$.

$$
\left|H_{2}(2)\right| \leq \frac{\alpha^{2}}{144}\left(\frac{17}{4}-\frac{\alpha}{4+\alpha^{2}}\right) .
$$

Proof. From the definition of the class $\mathcal{G}(\alpha)$ we can write

$$
1+\frac{z f^{\prime \prime}(z)}{f^{\prime}(z)}=1+\frac{1}{2} \alpha-\frac{\alpha}{2} \frac{1+\omega(z)}{1-\omega(z)} \quad\left(=1+\alpha-\alpha \frac{1}{1-\omega(z)}\right),
$$

where $\omega$ is analytic in $\mathbb{D}$ with $\omega(0)=0$ and $|\omega(z)|<1, z \in \mathbb{D}$. The last relation we can write in the form of

$$
\left(z f^{\prime}(z)\right)^{\prime}=\left[1-\alpha\left(\omega(z)+\omega^{2}(z)+\cdots\right)\right] f^{\prime}(z) .
$$

Putting $\omega(z)=c_{1} z+c_{2} z^{2}+\cdots$ in (2.13) and comparing the coefficients on $z, z^{2}, z^{3}$, after some simple calculations, we obtain

$$
\begin{aligned}
& a_{2}=-\frac{\alpha}{2} c_{1}, \\
& a_{3}=-\frac{\alpha}{6}\left[c_{2}+(1-\alpha) c_{1}^{2}\right], \\
& a_{4}=-\frac{\alpha}{24}\left[2 c_{3}+(4-3 \alpha) c_{1} c_{2}+\left(\alpha^{2}-3 \alpha+2\right) c_{1}^{3}\right] .
\end{aligned}
$$

From (2.14) we have, after some transformations,

$$
H_{2}(2)=\frac{\alpha^{2}}{144}\left[6 c_{1} c_{3}+(4-\alpha) c_{1}^{2} c_{2}-\left(\alpha^{2}+\alpha-2\right) c_{1}^{4}-4 c_{2}^{2}\right],
$$


and from here

$$
\left|H_{2}(2)\right| \leq \frac{\alpha^{2}}{144}\left[6\left|c_{1}\right|\left|c_{3}\right|+(4-\alpha)\left|c_{1}\right|^{2}\left|c_{2}\right|-\left(\alpha^{2}+\alpha-2\right)\left|c_{1}\right|^{4}+4\left|c_{2}\right|^{2}\right] .
$$

As in the proof of previous two theorems, we may suppose that $c_{1} \geq 0$. In that case the relations (2.6) are valid and by using the inequality first for $c_{3}$, after that for $c_{2}$, from (2.15) we have (we omit the details):

$$
\left|H_{2}(2)\right| \leq \frac{\alpha^{2}}{144}\left[4+(2-\alpha) c_{1}^{2}-\left(4+\alpha^{2}\right) c_{1}^{4}\right] .
$$

For $c_{1}^{2}=\frac{2-\alpha}{2\left(4+\alpha^{2}\right)}$ the function in the brackets in (2.16) has its maximum, and after calculation we have the statement of the theorem.

Especially for $\alpha=1$ we obtain the next implication

$$
\operatorname{Re}\left[1+\frac{z f^{\prime \prime}(z)}{f^{\prime}(z)}\right]<\frac{3}{2} \quad(z \in \mathbb{D}) \quad \Rightarrow \quad\left|H_{2}(2)\right| \leq \frac{9}{320} .
$$

The result is sharp for $\alpha=1$ due to $\omega(z)=z \frac{c_{1}-z}{1-c_{1} z}$ and $c_{1}=\frac{1}{\sqrt{10}}$.

In their paper [1] Bello and Opoola considered the class $S^{\star}(q)$ of functions $f \in \mathcal{A}$ satisfying the condition

$$
\frac{z f^{\prime}(z)}{f(z)}<\sqrt{1+z^{2}}+z \equiv q(z),
$$

They find that $\left|H_{2}(2)\right| \leq \frac{39}{48}$. In the next theorem we give the sharp result.

THEOREM 2.6. Let $f(z)=z+a_{2} z^{2}+a_{3} z^{3}+\cdots$ belongs to the class $S^{\star}(q)$. Then we have the next sharp estimation:

$$
\left|H_{2}(2)\right| \leq \frac{1}{4}
$$

Proof. First, by the definition of the class $S^{\star}(q)$, we have that

$$
\frac{z f^{\prime}(z)}{f(z)}=\sqrt{1+\omega^{2}(z)}+\omega(z)
$$

where $\omega$ is analytic in $\mathbb{D}$ with $\omega(0)=0$ and $|\omega(z)|<1, z \in \mathbb{D}$. Under the same notations as in previous three theorems, the authors in [1] obtained that

$$
H_{2}(2)=\frac{1}{3}\left(c_{1} c_{3}+\frac{1}{4} c_{1}^{2} c_{2}-\frac{7}{16} c_{1}^{4}-\frac{3}{4} c_{2}^{2}\right)
$$

If we apply the same method as in previous three cases, we easily obtain that

$$
\left|H_{2}(2)\right| \leq \frac{1}{3}\left(\frac{3}{4}-\frac{1}{4} c_{1}^{2}-\frac{1}{16} c_{1}^{4}\right) \leq \frac{1}{4} .
$$

The result is the best possible as the function $f_{q}$ defined by the condition

$$
\frac{z f_{q}^{\prime}(z)}{f_{q}(z)}=\sqrt{1+z^{4}}+z^{2}
$$

shows (i.e for $\omega(z)=z^{2}$ ).

\section{REFERENCES}

[1] Bello, R., AND Opoola, T. Upper bounds for fekete-szego functions and the second hankel determinant for a class of starlike functions. IOSR Journal of Mathematics 12, 2 (2017), 34-39.

[2] DuREn, P. L. Univalent functions, vol. 259 of Grundlehren der Mathematischen Wissenschaften [Fundamental Principles of Mathematical Sciences]. Springer-Verlag, New York, 1983.

[3] Kargar, R., And Ebadian, A. Ozaki's conditions for general integral operator. Sahand Communications in Mathematical Analysis 5, 1 (2017), 61-67. 
[4] Nunokawa, M., Saitoh, H., Ikeda, A., Koike, N., And Ota, Y. On certain starlike functions. No. 963. 1996, pp. 74-77. Univalent functions and the Briot-Bouquet differential equations (Japanese) (Kyoto, 1996).

[5] Obradovich, M., Ponnusami, S., and Virts, K.-U. Characteristics of the coefficients and partial sums of some univalent functions. Sibirsk. Mat. Zh. 54, 4 (2013), 852-870.

[6] Ozaki, S. On the theory of multivalent functions. II. Sci. Rep. Tokyo Bunrika Daigaku. Sect. A 4 (1941), 45-87.

[7] Prokhorov, D. V., And Szynal, J. Inverse coefficients for $(\alpha, \beta)$-convex functions. Ann. Univ. Mariae Curie-Skłodowska Sect. A 35 (1981), 125-143 (1984).

[8] SAKaguchi, K. A property of convex functions and an application to criteria for univalence. Bull. Nara Univ. Ed. Natur. Sci. 22, 2 (1973), 1-5.

[9] Singh, R., AND Singh, S. Some sufficient conditions for univalence and starlikeness. Colloq. Math. 47, 2 (1982), 309-314 (1983).

[10] Sokól, J., AND Thomas, D. K. The second Hankel determinant for alpha-convex functions. Lith. Math. F. 58, 2 (2018), 212-218.

[11] Thomas, D. K., Tuneski, N., And Vasudevarao, A. Univalent functions, vol. 69 of De Gruyter Studies in Mathematics. De Gruyter, Berlin, 2018. A primer.

[12] Umezawa, T. Analytic functions convex in one direction. F. Math. Soc. Japan 4 (1952), 194-202.

Open Access statement. This is an open-access article distributed under the terms of the Creative Commons AttributionNonCommercial 4.0 International License (https://creativecommons.org/licenses/by-nc/4.0/), which permits unrestricted use, distribution, and reproduction in any medium for non-commercial purposes, provided the original author and source are credited, a link to the CC License is provided, and changes - if any - are indicated. 